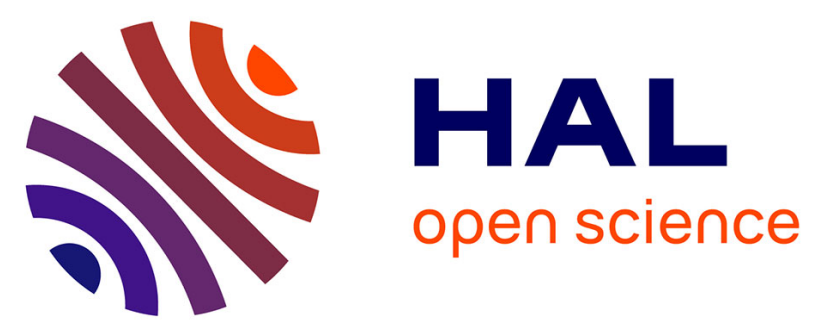

\title{
A Collision Avoidance Algorithm Based on the Virtual Target Approach for Cooperative Unmanned Surface Vehicles
}

Marco Bibuli, Gabriele Bruzzone, Massimo Caccia, Lionel Lapierre, Enrica Zereik

\section{To cite this version:}

Marco Bibuli, Gabriele Bruzzone, Massimo Caccia, Lionel Lapierre, Enrica Zereik. A Collision Avoidance Algorithm Based on the Virtual Target Approach for Cooperative Unmanned Surface Vehicles. MED: Mediterranean Conference on Control and Automation, Jun 2014, Palermo, Italy. pp.746-751, 10.1109/MED.2014.6961463 . lirmm-01904368

\section{HAL Id: lirmm-01904368 \\ https://hal-lirmm.ccsd.cnrs.fr/lirmm-01904368}

Submitted on 30 Oct 2018

HAL is a multi-disciplinary open access archive for the deposit and dissemination of scientific research documents, whether they are published or not. The documents may come from teaching and research institutions in France or abroad, or from public or private research centers.
L'archive ouverte pluridisciplinaire HAL, est destinée au dépôt et à la diffusion de documents scientifiques de niveau recherche, publiés ou non, émanant des établissements d'enseignement et de recherche français ou étrangers, des laboratoires publics ou privés. 


\title{
A Collision Avoidance Algorithm Based on the Virtual Target Approach for Cooperative Unmanned Surface Vehicles
}

\author{
Marco Bibuli, Gabriele Bruzzone, Massimo Caccia and Lionel Lapierre
}

\begin{abstract}
This paper presents a collision avoidance algorithm based on the virtual target path-following guidance technique, developed for Unmanned Surface Vehicle multiagent frameworks. The proposed collision avoidance procedure is integrated with the distributed guidance module already developed for cooperative and coordinated navigation of USVs. A basic integration of the collision avoidance system with the "Rules of the Road" is proposed too. Results of the overall collision free cooperative system are then presented.
\end{abstract}

\section{INTRODUCTION}

In the last years, the research interest of the marine robotics community has focused on the study and development of multi-agent systems, where a number of Unmanned Surface Vehicles (USVs) cooperates in a common operative frameworks, coordinating their motion, in order to achieve a global mission goal.

When two or more autonomous vehicles work in cooperation in the same operative area, the problem of vehicles collision has to be faced. Even if the mission is planned and cleared of any conflict between robots, it may happen that, due to external disturbances, different dynamic and kinematic characteristics, unpredicted conditions, online operation replanning, vehicles can come to a collision. The integration of a reactive and robust collision avoidance procedure within the automatic guidance architecture then becomes a natural choice.

The choice and implementation of an efficient collisionavoidance technique depends also on the sensing capabilities that each robot has with respect to the operating framework and to other entities belonging to the system. Positions of possible colliding vehicles can be detected through measurement coming from exteroceptive sensors like cameras, radars, range-meters; however, when a number of robots cooperates in a common framework, they are usually communicating a set of basic navigation information, such as positions, speed, direction, that can be shared to enhance the knowledge of the framework.

In literature, it is possible to find a number of papers describing multi-vehicle systems (a general description can be found in [1]) integrated with procedures and/or control laws to deal with the problem of inter-robot collision. Work [2] is focused on the control of two follower vehicles tracking a reference

M. Bibuli, G. Bruzzone and M. Caccia are with the Istituto di Studi sui Sistemi Intelligenti per l'Automazione, Consiglio Nazionale delle Ricerche, 16149 Genova, Italy marco.bibuli@ge.issia.cnr.it

L. Lapierre is with the Laboratoire d'Informatique, de Robotique et de Microlectronique de Montpellier, Centre National de la Recherche Scientifique, 161 rue Ada, 34392 Montpellier Cedex 5, France lapierredirmm.fr robot; the Virtual Robot control technique is introduced and through an appropriate use of its convergence properties, a global collision-free motion is achieved. In [3] the so called $l-l$ method, based only on local sensor-based information, is developed to achieve a collision-free leader-follower motion; the control law development relies on the feedback linearization technique, used to exponentially stabilize the relative distance and orientation of the followers. The methodologies described in the aforementioned papers are improved by the work [4], where the Virtual Robot and $l-l$ approaches are integrated in a reactive scheme based framework, proving the advantages of the method through the application to a three robot system. An alternative approach to achieve collision avoidance among multiple mobile robots is proposed in [5] where, based on the definition of velocity obstacles, sufficient conditions are derived for collision-free motion by reducing the problem to solving a low-dimensional linear program.

This work is focused on the development of a collision avoidance algorithm based on the virtual target approach and relying only on the position knowledge of the robots in the operative frameworks, to be integrated with the coordination guidance system, already developed and described in [6]. The virtual target approach offers a simple and robust methodology to achieve the collision avoidance task only on the basis of the position knowledge of the vehicles involved in the cooperation. A preliminary analysis of the possible integration of the collision avoidance technique with a behavior compliant with the "Rules of the Road" (COLREGS, see [7]) is also addressed in this paper.

In section II a brief description of multi-vehicle cooperative frameworks is given, focusing on Coordinated PathFollowing and Wingman problems. Section III reports the development details of the proposed virtual target based collision avoidance technique, while the integration with the COLREGS rules is reported in section IV. Results are proposed in section $\mathrm{V}$, finally reporting conclusions in section VI.

\section{CoOperative Frameworks}

This work focuses on two main cooperative frameworks for coordinated path-following, where the integration of the collision avoidance procedure will guarantee the safeness of the overall system.

A first case is the so called Coordinated Path-Following framework where two (or more) fully autonomous robots have to coordinate their motion, in order to perform a PathFollowing task along a predefined reference path, maintaining a fixed position configuration, i.e. a certain distance 
that can be set and updated online by the human operator. Vehicles know in advance the reference path they have to follow, and they share a few basic navigation information that are used to coordinate their motion to reach the desired position configuration.

When the goal is to require vehicles to move along different predefined paths, the Wingman Problem arises. Obviously in this case, the global task of cooperation has to be consistent: for instance, it is senseless to require the coordination of vehicle moving on a straight line with another one moving on a circular path. But it is possible, for instance, to imagine an operative framework where an USV is required to navigate along a straight path, coordinating its motion with another USV following a sinusoidal curve (evolving in the same direction of the straight line). In order to maintain, for example, a direct video link or consistency between heterogeneous environmental samplings, the vehicles have to coordinate their motion to navigate in an almost parallel lateral position configuration, one with respect to the other.

The idea at the basis of the coordination task relies on a further use of the virtual target approach, which is at the basis of the single-vehicle path-following addressed in [8] and used in this work as a base for the guidance system development of each vehicle, to compute relative positions between vehicles, thus properly regulating the relative speeds of advance in order to achieve the coordinated motion goal. Moreover, the virtual target based approach allows to completely uncouple the path-following task from the coordination one, thus maintaining unaltered the path-following algorithm and simply integrating a speed adaptation control law.

\section{A. Single-Vehicle Path-Following}

The single-vehicle path-following guidance system relies on the definition of a kinematic error system with respect to the Serret-Frenet frame $\langle v\rangle$, depicted in Figure 1:

$$
\left\{\begin{array}{l}
\dot{s}_{1}=-\dot{s}\left(1-c_{c} y_{1}\right)+U \cos \beta \\
\dot{y}_{1}=-c_{c} \dot{s} s_{1}+U \sin \beta \\
\dot{\beta}=r-c_{c} \dot{s}
\end{array}\right.
$$

where $s_{1}$ and $y_{1}$ are the longitudinal and lateral position errors respectively, $\beta$ is the orientation error, $U$ is the total velocity of the vehicle, $c_{c}$ is the local curvature of the path, $\dot{s}$ is the speed of the frame $\langle v\rangle$ along the path. Thus the following suitable yaw-rate control signal is generated through Lyapunov technique:

$$
r^{*}=\dot{\varphi}-k_{1}(\beta-\varphi)+c_{c} \dot{s}
$$

which drives the vehicles towards the related virtual target, attached to the frame $\langle v\rangle$, lying on the reference path; $k_{1}$ is a tunable gain, while $\varphi\left(y_{1}\right)=-\psi_{a} \tanh \left(k_{\varphi} y_{1}\right)$ is a variable approach angle, function on the lateral error $y_{1}$. At the same time a virtual speed regulation signal is computed and tuned through the $k_{2}$ parameter:

$$
\dot{s}^{*}=U \cos \beta+k_{2} s_{1}
$$

and applied to the virtual target, which will move towards the robot in order to reduce to zero the longitudinal error $s_{1}$ projected on its local reference frame. Details are extensively described in [8].

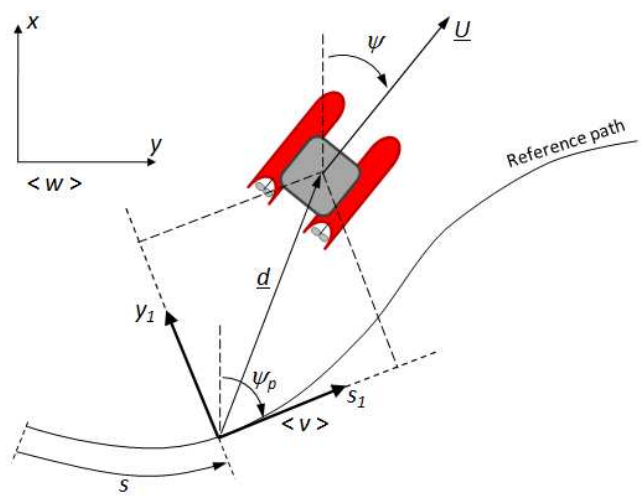

Fig. 1. Path-following framework

\section{B. Coordinated Path-Following}

The basic idea for the Coordinated Path-Following problem resolution is to decouple the task in two steps: $i$ ) first, each vehicle is forced to follow the desired path thanks to a path-following guidance system; ii) then, sharing the position on the related virtual targets, each vehicle regulates its surge speed in order to converge to and maintain the desired position configuration. With reference to Figure 2, for a two-vehicles framework the curvilinear distances are defined as $\Delta s_{1}=s_{2}-s_{1}$, for Vehicle-1, and $\Delta s_{2}=s_{1}-s_{2}$, for Vehicle-2. Defining the distance error for each vehicle as $e_{s_{i}}=\Delta s_{i}-D^{*}$, with $i=1,2$ and $D^{*}$ as the desired curvilinear distance between the vehicles, a smooth regulation law for the surge speed adaptation can obtained thanks to:

$$
u_{i}^{*}=C+\left(C-u_{\min }\right) \tanh \left(k_{u} e_{s_{i}}\right) \quad, \quad i=1,2
$$

with $C=\frac{u_{\max }+u_{\min }}{2}$ and where $u_{\min }$ and $u_{\max }$ are used-defined speed limits and $k_{u}$ is a controller gain.

Further details on multi-vehicle coordinated path-following

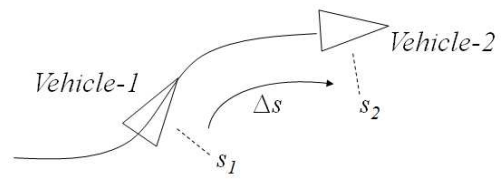

Fig. 2. Coordinated Path-Following curvilinear distance definition

are reported in [6].

\section{Wingman Problem}

In a Wingman problem framework, aeronautical term that originally refers to the plane flying beside the leader one in an aircraft formation [9], the task is to coordinate the motion of the vehicles that, in this case, can move along different paths. The wingman problem does not require a complete parallelism of the reference paths, but it is worth noticing that of course the desired paths have to be "oriented in the 
same direction" (Figure 3), in order to make the coordinated motion task feasible. To achieve the coordination task, i.e. to

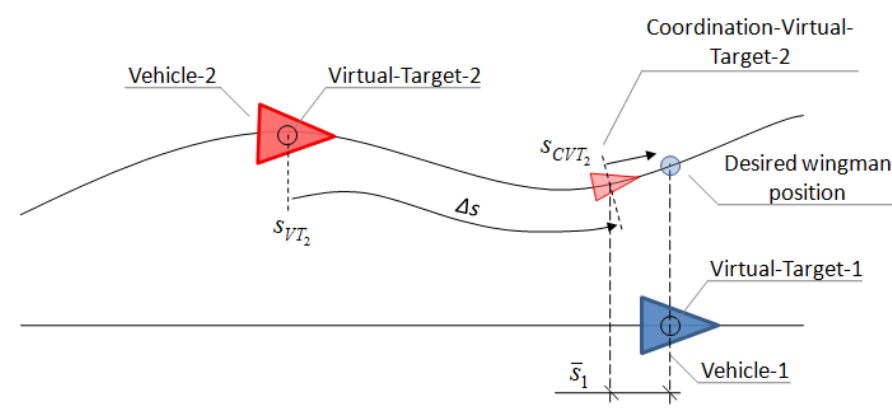

Fig. 3. General wingman problem framework

maintain a parallel vehicle formation, the key idea is to add a new virtual target, namely a Coordination Virtual Target (CVT) for each wingman vehicle. With reference to Figure 3 , the CVT is then forced to converge to the side of the leader vehicle, i.e. zeroing the $\bar{s}_{1}$ error referred to Vehicle-1 frame; computing and applying the CVT curvilinear speed

$$
\dot{s}_{C V T_{2}}^{*}=U_{1} \cos \bar{\beta}-\bar{k}_{2} \bar{s}_{1}
$$

it basically acts as a virtual target for Vehicle-1, moving on the path of Vehicle-2. $U_{1}$ is the speed of Vehicle- $1, \bar{\beta}$ is the difference between the local path tangents given by the position of the CVT and the virtual target of Vehicle-1, $\bar{s}_{1}$ is the projected longitudinal error, and $\vec{k}_{2}$ is a tunable gain. The CVT is then used to compute the curvilinear distance between itself and the virtual target of Vehicle-2, obtaining in this way the formation position error $\Delta s=s_{C V T_{2}}-s_{V T_{2}}$. The formation coordination task is achieved applying equation

$$
u_{2}^{*}=u_{1}+k_{u} \tanh \left(k_{e} \Delta s\right)
$$

to regulate the surge speed of Vehicle-2; $u_{1}$ is the feedforwarded speed of Vehicle-1, while $k_{u}$ and $k_{e}$ are tunable gains.

\section{Collision Avoidance}

A practical problem that arises when two or more autonomous vehicle are cooperating within the same framework, is that, due to initial conditions, kinematic and dynamic constraints, required task and etc, the vehicles can come to collide.

The simplest operative case that can be taken as an example is the one with the two vehicles following a straight path and maintaining a certain desired distance $D^{*}=\bar{D}$; at a certain time, the user requires the vehicles to exchange their positions, i.e. simply changing the sign of the desired distance, $D^{*}=-\bar{D}$. The effect is that the vehicle in the frontward position will slow down its speed, while the backward one will increase its surge, to converge both to the new desired positions. The effect of such operation is that, being the coordination guidance system only acts on the surge speed and, moreover, having no other shared information regarding for instance the position of the vehicles, there is no way of avoiding the collision between the robots.
For such a reason, to make the overall coordination guidance law intrinsically robust and safe, a basic cooperative collision avoidance algorithm has to be integrated within the guidance system.

The basic idea is to share the $x$ and $y$ positions between the robots, using such information to induce an avoidance reaction, if one vehicle is close to the other. A virtual safety circle is built around each vehicle and if a circle is intruded by another vehicle, such circle is used as a path reference for the intruding robot. Thus the intruding vehicle will locally follow the collision-avoiding circle path until its intrusion in the circle will be zeroed and its way to the original reference path will be free of collisions.

A generic collision avoidance situation is depicted in Figure 4; while Vehicle-1 is not intruding the safety circle of Vehicle-2, it tracks the approach angle computed by the Path-Following guidance system, converging to the virtual target on the reference path. The "intrusion" is defined as $2 \bar{R}-\left\|P_{1}-P_{2}\right\|$, where $\bar{R}$ is the user-defined radius of the safety circles, $P_{1}$ and $P_{2}$ are the position vectors of the vehicles with respect to a world fixed frame, see Figure 4(a). As Vehicle-1 intrudes the safety circle of Vehicle-2, a local virtual target is switched on the safety circle of Vehicle-2, forcing Vehicle-1 to turn in order to converge to the circle tangent (Figure 4(b)), at the point given by:

$$
P_{L T}=P_{2}+\bar{R}\left[\begin{array}{c}
\cos (\alpha) \\
\sin (\alpha)
\end{array}\right]
$$

with $\alpha=\operatorname{atan} 2\left(P_{2_{y}}-P_{1_{y}}, P_{2_{x}}-P_{1_{x}}\right)$.

The local reference tangent is then chosen between the two angles $\psi_{1}=\alpha-\frac{\pi}{2}$ and $\psi_{2}=\alpha+\frac{\pi}{2}$, as

$$
\psi_{L T}=\min \left(\left|\psi_{e}-\psi_{1}\right|,\left|\psi_{e}-\psi_{2}\right|\right)
$$

where $\psi_{e}$ is the direction of motion of Vehicle-1. Such tangent selection means to choose the closest direction to avoid the collision. A different choice can be integrated, for instance to comply with the "Rules of the Road", as it will be explained in the follow.

Vehicle-1 is then forced to track the safety circle while its "obstruction" is detected inside the safety circle, Figure 4(c). The obstruction is defined as the intersection between the circle and the original approach line, computed by the pure Path-Following algorithm, connecting Vehicle-1 and its virtual target on the reference path, as if no collisions had to be avoided. The obstruction value can be easily computed posing $d=[\cos (\gamma) \sin (\gamma)]^{T}$, where $\gamma$ is the angle of the original approach line; then, defining $E=P_{1}-P_{2}$, the discriminant for the calculation of the intersection can be computed by $\Delta=\left(d^{T} E\right)^{2}-d^{T} d\left(E^{T} E-\bar{R}^{2}\right)$, and if $\Delta>0$ the two intersection are given by:

$$
t_{1,2}=\frac{-d^{T} E \pm \sqrt{\Delta}}{d^{T} d}
$$

The obstruction value is evaluated as maximum intersection value greater than zero, $t=\max \left(0, t_{1}, t 2\right)$. When the obstruction value is zeroed, i.e. Vehicle- 1 has a free manoeuvring space, the virtual target is switched back on the original 
reference path, letting the vehicle to converge to it, as in Figure 4(d). Practically the condition can be relaxed to avoid numerical chattering (due to position measurement errors), posing the obstruction to be lower than a predefined threshold to exit from the collision avoidance procedure, $t<\bar{t}$. Moreover, it is worth noticing that, being the collision

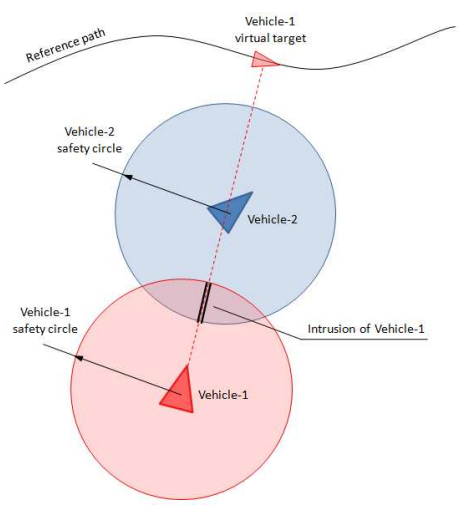

(a)

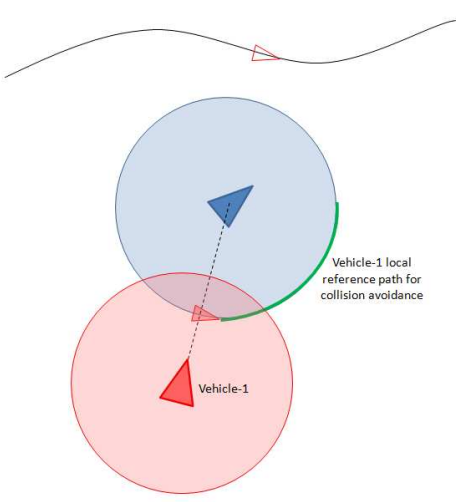

(b)

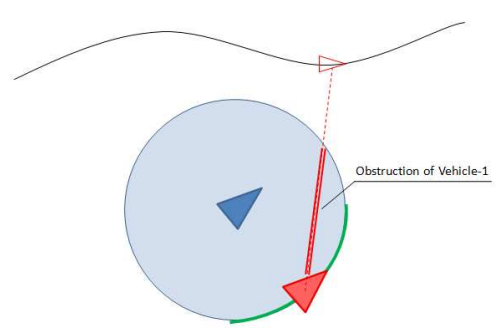

(c)

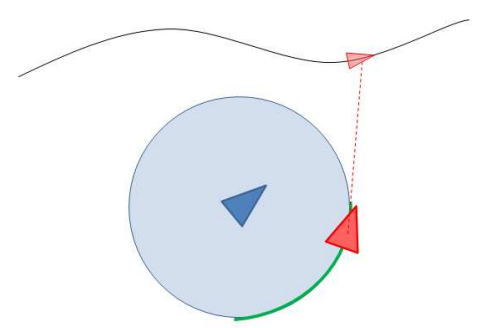

(d)

Fig. 4. Collision avoidance algorithm

avoidance routine integrated with the guidance system of all the vehicles belonging to the multi-agent framework, each vehicle cooperates in the avoidance manoeuvre as it will explained in the results' section.

\section{INTEGRATION OF THE COLREGS}

As introduced in the previous section, a compliant behavior with the COLREGS - "Rules of the Road" can be achieved forcing a desired turning direction, instead of leaving the system to automatically choose the avoidance action in terms of "closest direction" to reach.

A brief recall of the basic COLREGS rules for traffic regulation of power-driven vessels is given in order to address the problem:

1) Overtaking - An overtaking vessel must keep well clear of the vessel being overtaken.

2) Head-on situations - When two power-driven vessels are meeting head-on both must alter course to starboard so that they pass on the port side of the other.

3) Crossing situations - When two power-driven vessels are crossing, the vessel which has the other on the starboard side must give way.

4) The give-way vessel - The give-way vessel must take early and substantial action to keep well clear.

5) The stand-on vessel - The stand-on vessel may take action to avoid collision if it becomes clear that the give-way vessel is not taking appropriate action.

A simple improvement to the basic collision avoidance procedure is to substitute equation (8) with $\psi_{L T}=\psi_{1}$; this substitution has as results the choice of the tangent to the safety circle such that the colliding vehicle will avoid the collision performing an escape manoeuvre on the starboard direction. This kind of behavior makes the collision avoidance algorithm compliant with Head-on and Crossing situations, thus allowing vehicles to avoid path-crossing and giving the way complying with the rules.

Investigations on further improvements to introduce new behaviors to comply with the other rules of navigation are actually under evaluation.

\section{RESULTS}

The proposed results are performed simulating a number of CNR-ISSIA Charlie USVs (see [10]), navigating in different scenarios of cooperation. Experimental trials will be carried out (and will be reported in the final version of the paper) as soon as the development of the new CNR-ISSIA SWAP USVs (small vehicles designed for very shallow water applications) will be completed; such vehicles will be employed in cooperation with the Charlie USV to validate the proposed approach through experimental proof.

In Figure 5 a collision avoidance procedure is performed during an overtaking phase. The vehicles are initially following the straight path maintaining a predefined distance $D^{*}=20$ $\mathrm{m}$. Then the desired position configuration is changed to $D^{*}=-20 \mathrm{~m}$, i.e requiring the vehicles to switch their positions. The vehicle in the forward position will slow down its speed, while the backward vehicle will increase its surge, both converging to the new reference positions; as the vehicles get closer one to each other, the collision 
avoidance algorithm is triggered. First the backward Vehicle1 (red vehicle and line) intrudes the safety circle (the value of the safety circle radius has been set to $\bar{R}=6 \mathrm{~m}$ ) of forward Vehicle-2 (blue vehicle and line): this induces a collision avoidance reaction, forcing Vehicle-1 to turn, following the local circle path reference around Vehicle-2. As Vehicle-1 overtakes Vehicle-2, this last one enters in the Vehicle-2's safety circle, as a consequence it turns to avoid a possible collision, as it can be noticed in the middle of Figure 5. In this way, while performing an overtaking manoeuvre, both the vehicles cooperate to avoid the collision. Figure 6 highlights the speed profiles of vehicles while performing the overtaking action: Vehicle-1 has a higher speed value in the beginning, needed to reach and overtake the other vehicle, that is smoothly reduced as the target position is going to be reached; at the same time Vehicle- 2 has a reduced speed in the beginning, smoothly increased to reach the cruise speed, as the target position is approached.

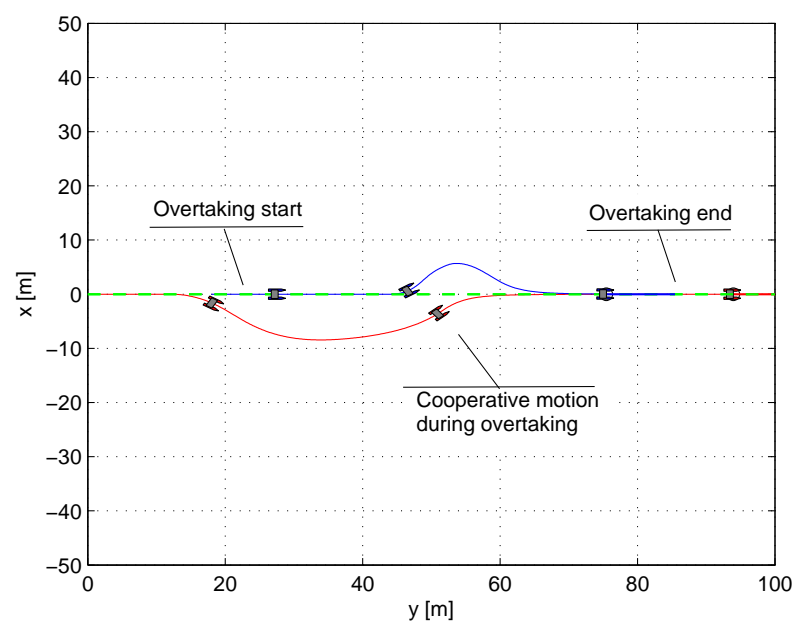

Fig. 5. Overtaking with collision avoidance experiment

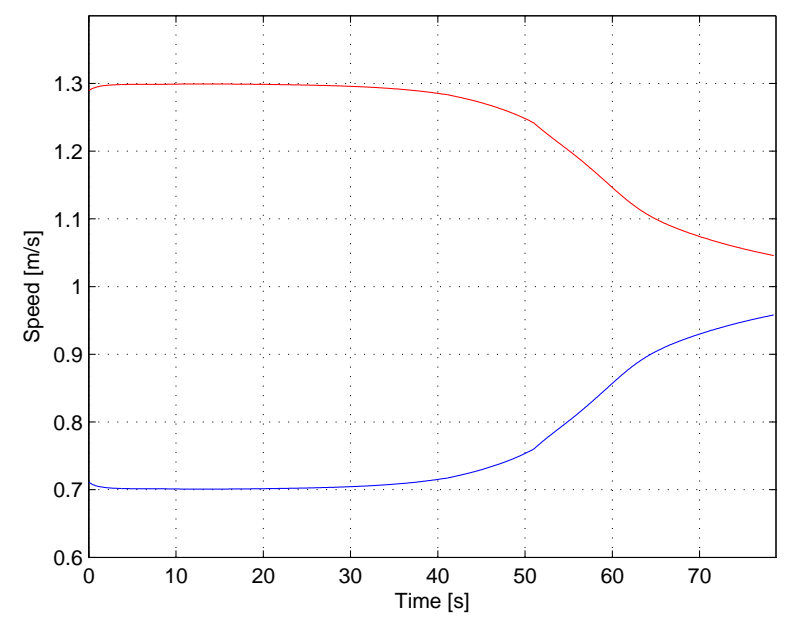

Fig. 6. Vehicles' speed profiles during overtaking
A Wingman framework is presented in Figure 7, where Vehicle-1 (red vehicle and line) has to follow a straight path, while Vehicle-2 (blue vehicle and line) follows the curved path while maintaining a parallel position with respect to Vehicle-1. When vehicles get closer to the crossing point the collision avoidance procedures is triggered. In this experiment the collision avoidance routine has been integrated with the COLREGS rules: being Vehicle-1 (red vehicle and line) on the starboard side of Vehicle-2 (blue vehicle and line), this last one is forced to steer on its starboard side to give way to Vehicle-1, as indicated by the rule (3). Once the collision has been avoided, Vehicle-2 converges again to its reference path and coordinates its motion, acting on the surge speed, to reach the parallel position on the side of Vehicle-1. The speed profiles of the vehicles are reported in Figure 8, where it is possible to notice that, while Vehicle1 maintains a constant cruise speed of $1 \mathrm{~m} / \mathrm{s}$, Vehicle-2 regulates its speed to reach and maintain the side position with respect to Vehicle-1. A minimum and maximum speed limits have been forced in order to maintain the speed values between 0.5 and $2.5 \mathrm{~m} / \mathrm{s}$. Figure 9 reports the absolute position distances of each vehicle with respect to the target to be tracked on the respective path. The error obviously diverges from zero when the collision avoidance procedure is triggered by each vehicle. The collision avoidance procedure

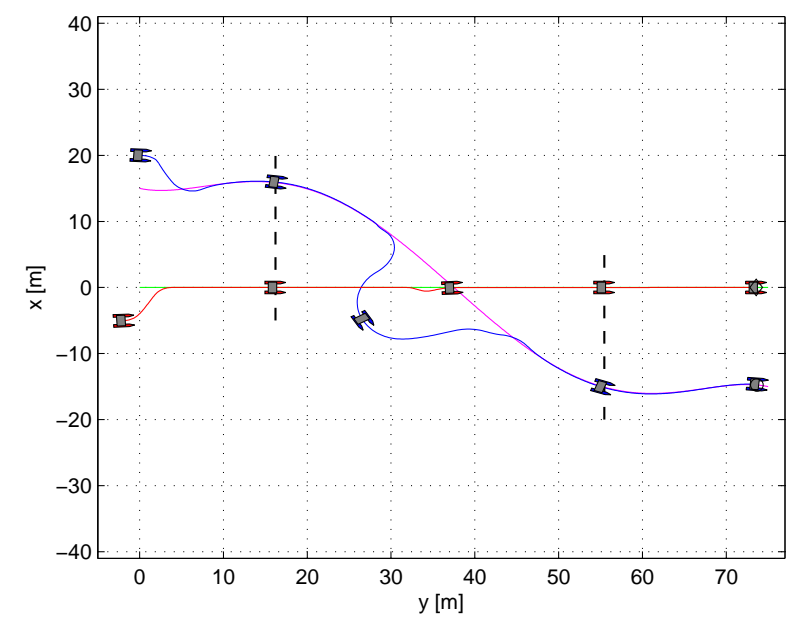

Fig. 7. Crossing with collision avoidance experiment, with COLREGS compliance

is extended to multi-vehicle frameworks (in the case of more than two vehicles) by cross-checking the distances between each couple of vehicles; the escape manoeuvre is computed and executed with respect to the closest colliding vehicle. A further investigation of possible conflicts and deadlocks using this approach is currently under study. An exemplificative scenario of a three vehicle crossing framework is reported in Figure 10.

\section{CONCLUSIONS}

The work proposes a simple and efficient procedure for collision avoidance between USVs working within a com- 


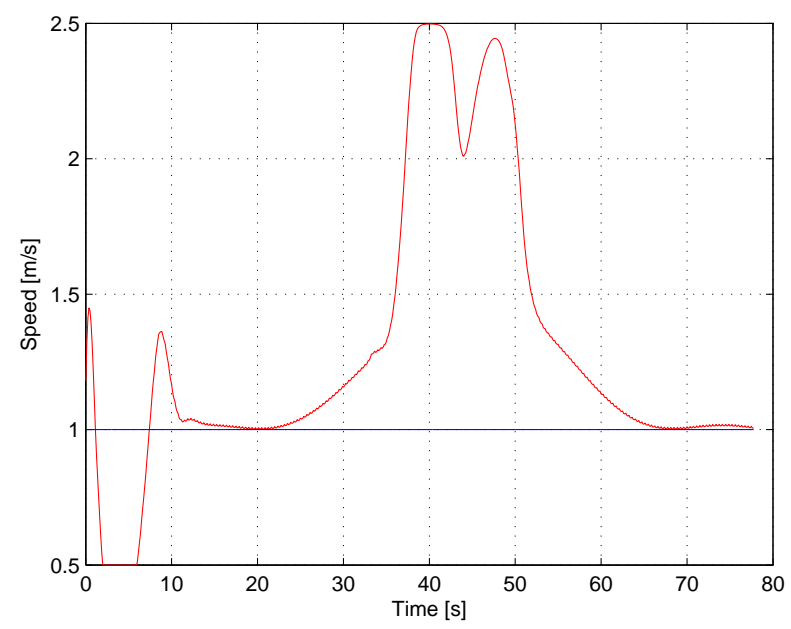

Fig. 8. Vehicles' speed profiles during crossing

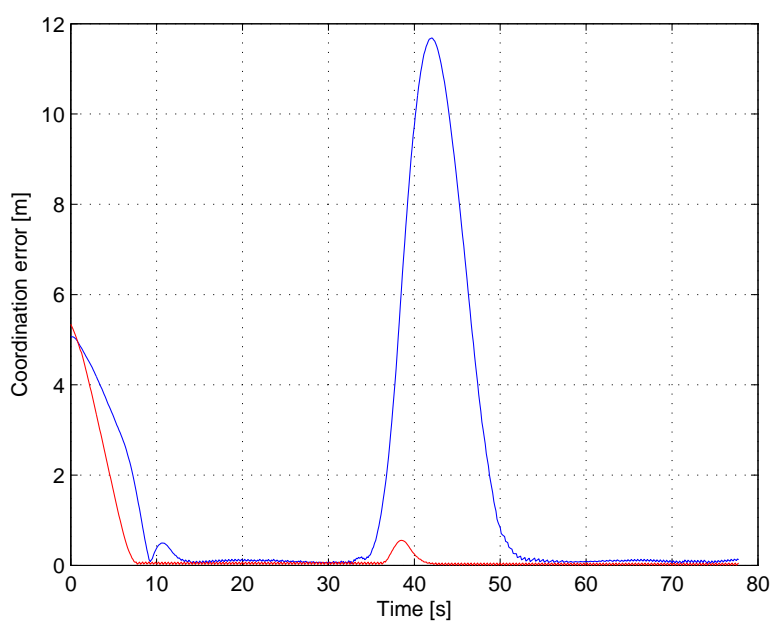

Fig. 9. Vehicles' absolute position errors with respect to the ideal position to be tracked on the path

mon cooperative framework. An integration of the "Rules of the Road" improves the basic avoidance routine, making the results attractive for the development of real systems that have to operate in general environment, usually characterized by manned vessel traffic.

The proposed virtual target based approach provides a simple methodology to manage the task of cooperating a set of vehicles and, at the same time, a simple and suitable collision avoidance module can be developed and integrated with the guidance system, only relying on the knowledge of other vehicles' positions.

Different topics have to be deeply investigated: how to manage and modify the formation configuration on-line, further studies and developments on the integration of the COLREGS rules, followed by simulations and experimentations with different number of vehicles, handling and distribution of the shared navigation data among the vehicles, needed to perform the cooperation tasks, analysis of possible conflicts

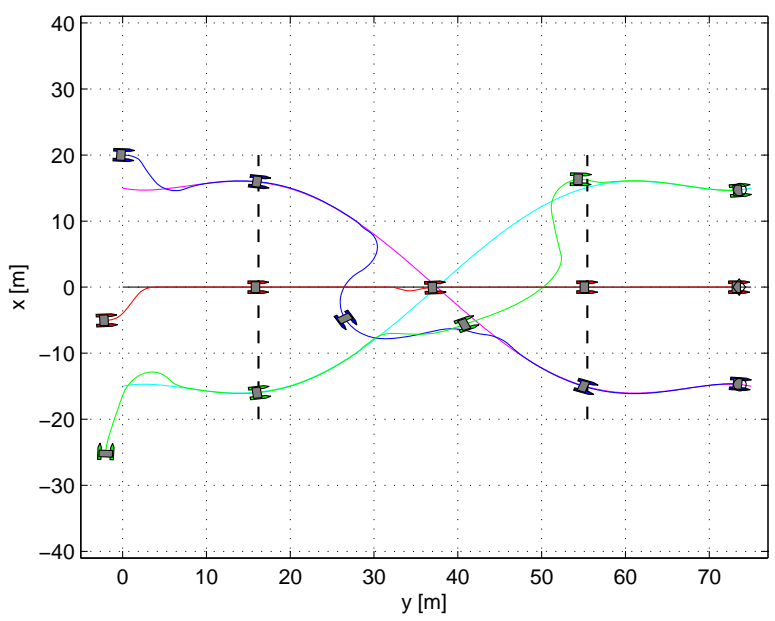

Fig. 10. Crossing experiment within a three vehicles framework

and/or deadlocks of the collision procedures in particular vehicle position configurations.

\section{ACKNOWLEDGMENTS}

This work has been funded by the bilateral agreement between CNR-ISSIA Genova, Italy and CNRS-LIRMM Montpellier, France for the years 2008-2009, within the research project "Coordinated mission control for autonomous marine vehicles".

\section{REFERENCES}

[1] J. Kalwa, "Final results of the European Project GREX: coordination and control of cooperating marine robots," in Proc. of 7th IFAC Symposium on Intelligent Autonomous Vehicles, 2010.

[2] J. Jongusuk and T. Mita, "Tracking control of multiple robots: a case study of inter-robot collision-free problem," Asian Journal of Control, vol. 4, no. 3, pp. 265-273, 2002

[3] J. P. Desai, J. Ostrowski, and V. Kumar, "Controlling formations of multiple mobile robots," in Proc. of the IEEE International Conference on Robotics and Automation, Leuven, Belgium, 1998.

[4] H. M. Ha, A. D. Nguyen, and Q. P. Ha, "Controlling formations of multiple mobile robots with inter-robot collision avoidance," in Proc. of the Australasian Conference on Robotics and Automation, Sydney, Australia, 2005

[5] J. Van Den Berg, S. J. Guy, M. Lin, and D. Manocha, "Optimal reciprocal collision avoidance for multi-agent navigation," in Proc. of the IEEE International Conference on Robotics and Automation, Anchorage (AK), USA, 2010.

[6] M. Bibuli, L. Lapierre, and M. Caccia, "Virtual target based coordinated path-following for multi-vehicle systems," in Proc. of 8th IFAC Conference on Control Applications in Marine Systems, 2010.

[7] M. Benjamin, J. Curcio, J. Leonard, and P. Newman, "Navigation of Unmanned Marine Vehicles in accordance with the rules of the road," in Proc. of the IEEE International Conference on Robotics and Automation, Orlando (FL), USA, May 2006.

[8] M. Bibuli, G. Bruzzone, M. Caccia, and L. Lapierre, "Path-following algorithms and experiments for an unmanned surface vehicle," Journal of Field Robotics, vol. 26, no. 8, pp. 669-688, 2009.

[9] S. Waydo, J. Hauser, R. Bailey, E. Klavins, and R. Murray, "UAV as a reliable wingman: A flight demonstration," IEEE Trans. on Control System Technology, vol. 15, no. 4, July 2007.

[10] M. Caccia, M. Bibuli, R. Bono, G. Bruzzone, G. Bruzzone, and E. Spirandelli, "Charlie, a testbed for usv research," in Proc. of MCMC 2009, $8^{\text {th }}$ Conference on Manoeuvring and Control of Marine Craft, Guaruja (SP), Brazil, September 2009. 